\title{
EDITORIAL
}

\section{The placenta in pre-eclampsia-Lymphangiogenesis and angiogenesis}

\section{Thajasvarie Naicker}

In the last decade, global maternal mortality has declined by $47 \%$, yet there is an estimate of 800 maternal deaths per day or 210 maternal deaths per 100000 live births. These pregnancy-related deaths is attributed to haemorrhage (35\%), pre-eclampsia/eclampsia (18\%), sepsis (8\%), unsafe abortion (9\%) and indirect causes including AIDS and malaria (18\%) [1]. Notably, resource poor countries account for 99\% of these deaths with 85\% occurring in Sub-Saharan Africa and Southern Asia [1]. Sub-Saharan Africa accounts for approximately $70 \%$ of people living with HIV infection globally [2]. In this region, South Africa is considered the epicenter of the global HIV pandemic. Off note, women represent approximately half of adults living with HIV infection; with majority occurring in women of reproductive age. Around 1.5 million HIV-infected women in Sub-Saharan Africa become pregnant each year [3]. In South Africa alone, approximately one-fifth of women in their reproductive age are HIV positive and recent HIV prevalence rates indicate that up to $22.8 \%$ of pregnant women are HIV-infected [4]. The province of KwaZulu-Natal has the highest prevalence of $16.9 \%$ amongst the South African provinces.

In the first trimester of normal pregnancy $(\mathrm{N})$, maternal spiral arteries undergo remodeling by invasive fetal cytotrophoblast cells. These cells migrate across two pathways, the interstitial pathway where they cross the decidua into the myometrium and the endovascular pathway where they migrate into the lumen of the spiral artery. In the decidua, adhesion molecules of the fetal cytotrophoblast transform the endothelium to an epithelial phenotype. This enables its physiological conversion into a large bore conduit that allows for the nourishment of the growing fetus. In pathological pregnancies such as preeclampsia, this transformation is incomplete. Pre-eclampsia (PE) is characterized by new onset high blood pressure $(>140 / 90 \mathrm{mmHg}$ ) and proteinuria ( $\geq 300 \mathrm{mg}$ in a $24 \mathrm{~h}$ urine sample or a dipstick result of $\geq$ $1+$ in the second half of pregnancy. In South Africa, maternal deaths due to hypertension in pregnancy is $14.8 \%$, of which $83 \%$ is attributed to pre-eclampsia. Within this high HIV endemic region, it is plausible that the immune insufficiency stimulated by HIV may impact the immune hyper-reactivity associated with pre-eclampsia. There is also a dearth of information whether a lymphatic system exists in the placenta. The role of placental angiogenic versus lymph-angiogenic factors in HIV associated preeclampsia warrants attention.

Our group has reported a reticular lymphatic-like conductive network within the placental stroma [5]. Lymphatic vessel endothelial receptor-1 (LYVE-1) immunoexpression was higher $(\mathrm{p}=0.0001)$ in the HIV+ cohort. However, irrespective of HIV status and pregnancy type, LYVE-1 was elevated in the conducting vs exchange villi $(\mathrm{p}=0.01)$. Within the placenta, podoplanin (PDPN) immunoexpression was up-regulated in the exchange vs conducting villi $(p=0.0001)$, irrespective of the pregnancy type and HIV status. Notably, PDPN was up-regulated in HIV+ vs HIV-groups regardless of pregnancy and villi type.

Based on HIV status, we report that soluble fms-like tyrosine kinase-1 (sFlt-1) immunoexpression were similar in exchange and stem villi $(\mathrm{p}=0.53)$. sFlt-1 immunoexpression was higher in exchange villi of PE vs $\mathrm{N}$ women $(\mathrm{p}=0.003)$. However, there was no interaction between HIV infection and pregnancy type for sFlt- 1 expression in both exchange and stem villi. There was no correlation between the exchange and stem villi immunoexpression of sFlt-1 (p-0.563) [6]. We also report no significant effect of HIV status on soluble Endoglin (sEng) immunoexpression within exchange villi $(\mathrm{p}=0.088)$. However, based on pregnancy type, sEng immunoexpression differed significantly within stem villi $(\mathrm{p}<0.001)$. Higher sEng immunoexpression was observed in the PE vs $\mathrm{N}$ groups for both villi types. No interaction between HIV status and pregnancy type on sEng immunoexpression in both exchange $(p=0.207)$ and stem villi $(p=0.421)$ was observed [7]. Immunoexpression of placental growth factor (PlGF) was lower within PE compared to $\mathrm{N}(\mathrm{p}=0.001)$, however, there was a significant downregulation of PIGF in the HIV+ compared to HIV-groups. Also, there was a significant effect of HIV status on immunoexpression of vascular endothelial growth factor (VEGF) in the exchange villi $(\mathrm{p}=0.005)$. VEGF immunoexpression was lower in PE vs N pregnancy across villi types [8].

Finally, the classical lymphatic vessel drainage is absent in the placenta. Moreover, whilst preeclampsia has an imbalance between pro- and anti-angiogenic factors, the neutralising effect of HIV infection on the immune system is insufficient to neutralise its development. Current studies include 
VEGF receptors, platelet endothelial cell adhesion molecule-1 and angiopoietin as they have a predictive test value for the early detection of the disease.

\section{REFERENCES}

1. Oyston, C., Rueda-Clausen, C.F. \& Baker, P.N. (2014). Current challenges in pregnancy-related mortality. Obstetrics, Gynaecology \& Reproductive Medicine. 24(6):162-169.

2. WHO (2016) http://www.who.int/mediacentre/factsheets/fs360/en

3. Chi, B.H., Stringer, J.S.A. \& Moodley, D. (2013). Antiretroviral Drug Regimens to Prevent Mother-To-Child Transmission of HIV: A Review of Scientific, Program, and Policy Advances for Sub-Saharan Africa Curr HIV/AIDS Rep 10: 124-133

4. UNAIDS (2015) http://www.unaids.org/en/regionscountries/countries/southafrica

5. Onankoy A. Onyangunga, Jagidesa Moodley, Vesselina Merhar, David A. Ofusori and Thajasvarie Naicker (2016). Lymphatic vascular endothelial hyaluronan receptor-1 immunoexpression in placenta of HIV infected pre-eclamptic women. Journal of Reproductive Immunology 117:81-88.

6. Nalini Govender, Jagidesa Moodley, Premjith Gathiram and Thajasvarie Naicker (2014). Soluble fms-like tyrosine kinase-1 in HIV infected pre-eclamptic South African Black women. Placenta 35: 618-624.

7. Nalini Govender, Thajasvarie Naickera and Jagidesa Moodley (2015). Endoglin in HIV associated preeclamptic placentae. Hypertension in Pregnancy 34(3):1-13.

8. N Govender, T Naicker and J Moodley (2013). Maternal imbalance between pro-angiogenic and antiangiogenic factors in HIV infected pre-eclamptics. Cardiovascular Journal of Africa 24(4):217-222.

Correspondence author: Thajasvarie Naicker

Optics and Imaging Centre, College of Health Sciences, University of KwaZulu-Natal, South Africa.

Research Journal of Health Sciences subscribed to terms and conditions of Open Access publication. Articles are distributed under the terms of Creative Commons Licence (CC BY-NC-ND 4.0). (http://creativecommons.org/licences/by-nc-nd/4.0).

http://dx.doi.org/10.4314/rejhs.v5i2.1 


\section{ÉDITORIAL}

\section{Le placenta dans la pré-éclampsie - Lymphangiogenèse et angiogenèse}

\section{Thajasvarie Naicker}

Au cours de la dernière décennie, la mortalité maternelle mondiale a diminué de $47 \%$, mais il existe une estimation de 800 décès maternels par jour ou 210 décès maternels pour 100000 naissances vivantes. Ces décès liés à la grossesse sont attribués à une hémorragie $(35 \%)$, une pré-éclampsie / éclampsie (18\%), une septicémie (8\%), un avortement à risque (9\%) et des causes indirectes, y compris le sida et le paludisme (18\%) [1]. Notamment, les pays pauvres en ressources représentent $99 \%$ de ces décès, dont $85 \%$ se produisent en Afrique subsaharienne et en Asie du Sud [1]. L'Afrique subsaharienne représente environ $70 \%$ des personnes vivant avec une infection par le VIH dans le monde [2]. Dans cette région, l'Afrique du Sud est considérée comme l'épicentre de la pandémie mondiale du VIH. Les femmes représentent environ la moitié des adultes vivant avec une infection par le VIH; Avec la majorité chez les femmes en âge de procréer. Environ 1,5 million de femmes infectées par le VIH en Afrique subsaharienne deviennent enceintes chaque année [3]. En Afrique du Sud seulement, environ un cinquième des femmes en âge de procréer sont des taux de prévalence du VIH récents et réels, ce qui indique que jusqu'à 22,8\% des femmes enceintes sont infectées par le VIH [4]. La province de KwaZulu-Natal a la plus forte prévalence de $16,9 \%$ parmi les provinces sud-africaines.

$\mathrm{Au}$ premier trimestre de grossesse normale $(\mathrm{N})$, les artères spirales maternelles subissent un remodelage par des cellules cytotrophoblastiques fœtales invasives. Ces cellules migrent à travers deux voies, la voie interstitielle où elles traversent la décidua dans le myomètre et la voie endovasculaire où elles migrent vers la lumière de l'artère spirale. À la décidua, les molécules d'adhésion du cytotrophoblaste fœetal transforment l'endothélium en un phénotype épithélial. Cela permet sa conversion physiologique en un conduit à gros orifices qui permet de nourrir le foetus en croissance. Dans les grossesses pathologiques telles que la pré-éclampsie, cette transformation est incomplète. La pré-éclampsie (PE) se caractérise par une nouvelle pression artérielle $(>140 / 90 \mathrm{mmHg}$ ) et une protéinurie $(=300 \mathrm{mg}$ dans un échantillon d'urine de $24 \mathrm{~h}$ ou un résultat de la jauge de $=1+$ au cours de la deuxième moitiéde la grossesse. L'Afrique, les décè s maternels dus à l'hypertension pendant la grossesse est de 14,8\%, dont $83 \%$ sont attribué s à la pré-é clampsie. Dans cette ré gion endé mique élevée du VIH, il est plausible que l'insuffisance immunitaire stimulée par le VIH puisse avoir une incidence sur l'hyperréactivitéimmunitaire associée à Pré-éclampsie. Il existe également une pénurie d'information sur l'existence d'un système lymphatique dans le placenta. Le rôle des facteurs angiogéniques placentaires contre les angiogènes lymphatiques dans la prééclampsie associée au VIH nécessite une attention particulière.

Notre groupe a rapporté un réseau conducteur de type lymphatique réticulaire dans le stroma placentaire [5]. L'immunothérapie exprimée par le récepteur endothélial des vaisseaux lymphatiques 1 (LYVE-1) était plus élevée $(\mathrm{p}=0,0001)$ dans la cohorte VIH +. Cependant, indépendamment du statut du VIH et du type de grossesse, LYVE-1 était élevé dans les villosités de conduite contre l'échange $(\mathrm{p}=0,01)$. Dans le placenta, l'immuno-expression de la podoplanine (PDPN) a été régulée dans l'échange contre la réalisation de villosités ( $\mathrm{p}=0,0001)$, quel que soit le type de grossesse et le statut VIH. Notamment, le PDPN était réglementé par les groupes VIH + vs VIH, indépendamment de la grossesse et du type des villosités.

En se basant sur le statut du VIH, nous signalons que l'immunothérapie soluble dans les fms-like tyrosine kinase-1 (sFlt-1) était similaire en échange et les villosités de la tige $(\mathrm{p}=0,53)$. L'immunothérapie de sFlt-1 était plus élevée en échange de villosités de PE contre $\mathrm{N}$ femmes $(\mathrm{p}=0,003)$. Cependant, il n'y avait aucune interaction entre l'infection à VIH et le type de grossesse pour l'expression de sFlt-1 dans les villosités d'échange et de tige. Il n'y avait pas de corrélation entre l'échange et l'immuno-expression des villosités de la tige de sFlt-1 (p-0.563) [6]. Nous ne signalons pas non plus d'effet significatif du statut VIH sur l'immuno-expression endogline soluble (sEng) dans les villosités d'échange $(\mathrm{p}=0,088)$. Cependant, selon le type de grossesse, l'immunothérapie à la sEng différait significativement dans les villosités de la tige $(\mathrm{p}<0,001)$. Une plus grande exposition immuno-immune a été observée dans les groupes PE contre $\mathrm{N}$ pour les deux types de villosités. Aucune interaction entre l'exposition au VIH et le type de grossesse sur l'immuno-expression de la sEng à la fois dans l'échange $(p=0,207)$ et les villosités de la tige $(p=0,421)$ a été observée [7]. L'exposition à l'immuno-expression du facteur de croissance placentaire (PlGF) était plus faible dans l'EP par rapport à $\mathrm{N}(\mathrm{p}=0,001)$, cependant, il y avait une régulation négative significative 
de PIGF dans le VIH + par rapport aux groupes de VIH. En outre, il y a eu un effet significatif du statut VIH sur l'immuno-expression du facteur de croissance endothélial vasculaire (VEGF) dans les villosités d'échange $(\mathrm{p}=0,005)$. L'immunothérapie à VEGF était plus faible chez la grossesse de PE contre $\mathrm{N}$ chez les types de villosités [8].

Enfin, le drainage classique des vaisseaux lymphatiques est absent dans le placenta. En outre, alors que la prééclampsie présente un déséquilibre entre les facteurs pro et anti-angiogéniques, l'effet neutralisant de l'infection par le VIH sur le système immunitaire est insuffisant pour neutraliser son développement. Les études actuelles comprennent les récepteurs de VEGF, la molécule d'adhésion aux cellules endothéliales plaquettes-1 et l'angiopoiétine car elles ont un test prédictif.

\section{REFERENCES}

1. Oyston, C., Rueda-Clausen, C.F. \& Baker, P.N. (2014). Current challenges in pregnancy-related mortality. Obstetrics, Gynaecology \& Reproductive Medicine. 24(6):162-169.

2. WHO (2016) http://www.who.int/mediacentre/factsheets/fs360/en

3. Chi, B.H., Stringer, J.S.A. \& Moodley, D. (2013). Antiretroviral Drug Regimens to Prevent Mother-To-Child Transmission of HIV: A Review of Scientific, Program, and Policy Advances for Sub-Saharan Africa Curr HIV/AIDS Rep 10: 124-133

4. UNAIDS (2015) http://www.unaids.org/en/regionscountries/countries/southafrica

5. Onankoy A. Onyangunga, Jagidesa Moodley, Vesselina Merhar, David A. Ofusori and Thajasvarie Naicker (2016). Lymphatic vascular endothelial hyaluronan receptor-1 immunoexpression in placenta of HIV infected pre-eclamptic women. Journal of Reproductive Immunology 117:81-88.

6. Nalini Govender, Jagidesa Moodley, Premjith Gathiram and Thajasvarie Naicker (2014). Soluble fms-like tyrosine kinase-1 in HIV infected pre-eclamptic South African Black women. Placenta 35: 618-624.

7. Nalini Govender, Thajasvarie Naickera and Jagidesa Moodley (2015). Endoglin in HIV associated preeclamptic placentae. Hypertension in Pregnancy 34(3):1-13.

8. N Govender, T Naicker and J Moodley (2013). Maternal imbalance between pro-angiogenic and antiangiogenic factors in HIV infected pre-eclamptics. Cardiovascular Journal of Africa 24(4):217-222.

Correspondence author: Thajasvarie Naicker

Optics and Imaging Centre, College of Health Sciences, University of KwaZulu-Natal, South Africa. 